Reprod. Nutr. Dévelop., 1980, 20 (6), 1767-1779.

\title{
Effet des ondes électromagnétiques non ionisantes sur la viabilité et l'hérédité des cellules de mammifères en culture établie
}

\author{
par M. GILLOIS, C. AUGE *, C. CHEVALET \\ Laboratoire de Génétique cellulaire, I. N. R. A., B. P. no 12. \\ 31320 Costonet-Tolosan, \\ * Laboratoire DERMO, Centre de recherches O. N. E. R. A. \\ 31055 Toulouse, Cedex, B. P. no 4025.
}

Summary. Effect of non-ionizing electromagnetic waves on the viability and heredify of mammalian cells in culture.

This paper describes an automatic control apparatus for studying the non-thermal effects of non-ionizing electromagnetic waves $(2450 \mathrm{MHz})$ on mammalian cells in culture. The cells are exposed in their culture medium, stuck on their usual support. The advantage of this apparatus is that somatic genetics techniques can be used.

Non-ionizing electromagnetic waves have a specific non-thermal, lethal effect ; they are not mutagenic and enhance the mutagenic ability of ethyl methare sulfonate.

\section{Introduction.}

Les expositions aux ondes électromagnétiques non ionisantes de fortes intensités ont des effets pathologiques différents des expositions chroniques aux faibles intensités. Les premières soit entraînent la mort par arrêt respiratoire ou cardiaque, soit provoquent de graves lésions par le réchauffement des tissus irradiés : brûlures, nécroses cellulaires, troubles dégénératifs des gonades, cataractes. Les secondes induisent des névroses associées à une dystonie neurocirculatoire, dans un cas un syndrome asthénovégétatif et en général une diminution d'activité sexuelle (Marha et al., 1971).

L'exposition chronique de mammifères, principalement rat, mais aussi souris ef lapin, fait apparaître des perturbations de comportement associées à des désordres des régulations neuro-endocrines (Baranski ef Edelwejn, 1975 ; Michaelson ef al., 1975 ; Presman, 1970). En particulier, les réflexes conditionnés sont inhibés, l'excitabilité est diminuée, l'activité motrice spontanée est augmentée. Ces caractéristiques sont concomitantes d'une diminution de l'activité cholinestérase dans la moëlle épinière, le cœur, le foie et la fraction sérique du sang et d'une augmentation de l'acétylcholine dans les hémisphères cérébraux ef la moëlle épinière (Marha ef al., 1971).

Des effets tératogènes ont été observés au cours du développement des pupes de Tenebrio molitor (Carpenter et Livstone, 1971 ; Lindauer ef al., 1974) ainsi que des 
modifications de la morphologie des cellules maintenues en culture (Lin et Chen, 1975 ; Sawicki et Ostrowski, 1968 ; Stodolinik-Barnaska, 1967 ; Valtonen, 1966 ; Chiabrera ef al., 1979 ; Alam et al., 1978). Enfin il a été montré que les ondes électromagnétiques non ionisantes n'avaient aucun pouvoir mutagène chez $E$. Coli (Averbeck et al., 1976 ; Berteaud ef al., 1975), la levure (Averbeck et al., 1976) ou bien les champignons comme Aspergillus nidulans (Baranski et al., 1976).

Actuellement, il n'existe pas d'interprétation générale cohérente commune à ces phénomènes. Les causes de cette situation sont les difficultés de l'analyse expérimentale. L'une d'entre elles est de pouvoir séparer sans ambiguïté les effets spécifiques improprement désignés non thermiques (Marha ef al., 1971) des ondes électromagnétiques non ionisantes des conséquences de leurs effets thermiques (Marha et al., 1971). Pour expliquer les effets spécifiques, les conjectures généralement admises font infervenir la sensibilité aux ondes électromagnétiques non ionisantes des électrons périphériques des ions $\mathrm{Ca}^{++}$situés au voisinage des surfaces des macromolécules. Sous l'influence d'un champ électromagnétique non ionisant ces électrons seraient délocalisés ef formeraient des dipôles induits, lesquels seraient à l'origine d'importants changements de configuration des macromolécules (Bawin et al., 1978 ; Fröhlich, 1973, 1977 ; Grodsky, 1975, 1976 ; Neumann et al., 1973 ; Neumann et Katchalsky, 1972). Ces conjectures insistent sur le mécanisme de l'amplification quantique (Fröhlich, 1978).

Pour infirmer ou affirmer de telles conjectures et leur donner un statut d'interprétation, il est nécessaire de trouver une cible intracellulaire dont la sensibilité aux ondes électromagnétiques non ionisantes met en évidence leurs effets spécifiques. Cette cible peut être l'ADN. L'effet des ondes électromagnétiques non ionisantes soit fait intervenir les mêmes mécanismes que ceux des ondes électromagnétiques ionisantes, soit dépend d'aufres mécanismes. Mais il est aussi possible que cette cible ne soit pas l'ADN, ce qui impose une toute autre approche de l'étude du mécanisme des effets des ondes électromagnétiques non-ionisantes. En utilisant des cellules en cultures établies de porc (PK 15) et de hamster ( $\mathrm{CHO}$ ), nous montrons l'existence d'un effet léłal spécifique des ondes électromagnétiques non-ionisantes, leur caractère non mutagène et leur propriété d'amplification du pouvoir mutagène de l'éthyl méthane sulfonate. Les résultats obtenus permettent d'envisager l'isolement de mutants cellulaires résistants ou sensibles aux ondes électromagnétiques non ionisantes.

\section{Matériel et méthodes.}

Les deux lignées cellulaires utilisées sont la lignée cellulaire $\mathrm{CHO}(\mathrm{CCL}$ 61) et la lignée PK 15 (CCL 63). La lignée cellulaire PK 15 a été isolée en 1955 à partir d'un rein de porc (Ruddle, 1961), la lignée CHO en 1957 à partir d'un ovaire de hamster (Puck ef al., 1958). La"durée moyenne d'un cycle cellulaire de PK 15 est de 19 à 20 h à $38^{\circ} \mathrm{C}$, et celle de $\mathrm{CHO}$ est de $16 \mathrm{~h}$ à $36^{\circ} \mathrm{C}$; ces températures sont respectivement les températures optimales de ces lignées cellulaires. Ces mesures ont été faites dans le milieu minimum de Eagle (MEM 0121, Eurobio), avec solution de Hanks, complémenté avec les acides aminés non essentiels $\left(1.10^{2} \mathrm{M}\right)$ et $4 \mathrm{p} .100$ de sérum embryon- 
naire de veau, de la pénicilline $(100 \mathrm{u} . \mathrm{i} . / \mathrm{ml})$, de la streptomycine $(100 \mu \mathrm{g} / \mathrm{ml})$, tamponné avec 1.10-2 $\mathrm{M}$ de tris-(hydroxymethyl) aminomethane (Merk). Dans ces conditions de culture les cellules $\mathrm{CHO}$ ont une efficacité d'étalement indépendante de leur densité. Le même milieu complémenté en thymidine $\left(1,510-{ }^{5} \mathrm{M}\right)$, en hypoxanthine $\left(1.10^{-4} \mathrm{M}\right)$ et en uridine $\left(5.10^{-5} \mathrm{M}\right)$ permet un clonage des cellules PK 15. Les manipulations ont été réalisées stérilement en boîte de plastique NUNC qui sont transparentes aux ondes électromagnétiques et dont la surface inférieure sur laquelle adhèrent les cellules est particulièrement plane. Après trypsination, 1000 cellules isolées sont étalées dans une boîte NUNC de $60 \mathrm{~cm}^{3}$ soit $25 \mathrm{~cm}^{2}$ de surface de culture, dans $5 \mathrm{ml}$ de milieu. Les colonies cellulaires issues de ces cellules isolées sont fixées, colorées au bleu de UNNA et complées après huit jours de culture à $36^{\circ} \mathrm{C}$ pour $\mathrm{CHO}$ et 9 à 10 jours à $38^{\circ} \mathrm{C}$ pour les cellules PK 15. Le nombre moyen de colonies décomptées divisé par le nombre moyen de cellules étalées dans la boîte est l'efficacité d'étalement. Le nombre est en général voisin de $2 / 3$ pour $\mathrm{CHO}$ et de $1 / 3$ pour $\mathrm{PK}$ dans ces conditions de culture. Le nombre moyen de colonies décomptées dans des boîtes NUNC ayant subi un traitement, divisé par le nombre moyen de colonies décomptées dans des boîtes NUNC non traitées est le taux de survie. Le nombre varie de 1 à $10^{-6}$ qui est la limite de détection imposée par les possibilités matérielles.

L'induction chimique de la mutagenèse est faite par un agent alkylant, l'Ethyl Méthane Sulfonate (EMS). Pendant $6 \mathrm{~h}, 10.10^{6}$ cellules, sont exposées à $300 \mu \mathrm{g} / \mathrm{ml}$ d'EMS. Puis les cellules sont lavées deux fois dans du Hanks ef remises dans le milieu de culture. Le temps d'expression des mutations est de 3 à 7 jours. La sélection des cellules mutantes se fait par étalement de $5.10^{5}$ cellules par boîte NUNC de $25 \mathrm{~cm}^{2}$, en présence de l'agent de sélection.

Les caractères de résistance sont la résistance à la thioguanine $\left(8.10^{-6} \mathrm{M}\right)$ ef la résistance à l'ouabaïne $\left(1.10^{-6} \mathrm{M}\right)$. Le taux maximum de mutation est atteint 2 jours après la mutagenèse pour la résistance à l'ouabaīne, et 4 jours après la mutagenèse pour la résistance à la thiognanine. Ces taux maximum se maintiennent en l'absence de sélection pendant les 20 jours qui suivent la mutagenèse. Le comptage des colonies résistantes à l'agent de sélection se réalise après 30 jours.

L'appareillage d'émission des ondes électromagnétiques non ionisantes se compose d'une source, d'une ligne d'amenée, d'un applicateur. La source est un générateur de puissance de $200 \mathrm{~W}$. Elle est stabilisée à une fréquence de $2450 \mathrm{MHz}$, sa puissance de sortie contrôlable varie de 0 à $50 \mathrm{~W}$. Un circulateur et coupleur permettant la mesure de la puissance y sont adjoints. Une ligne d'amenée, constituée d'un guide d'ondes ef d'une ligne coaxiale souple relie la source à l'applicateur.

L'applicateur est de type cavitaire, avec introduction de l'énergie par le bas et prise de la température par une sonde à thermistance, plongée dans le milieu de culture. La cavité est tapissée latéralement par un circuit réfrigérant en téflon et un absorbant. Le liquide réfrigérant est de l'eau provenant d'une source froide de 30 l à une température constante de $4^{\circ} \mathrm{C}$. Une pompe accélère le débit. La boîte NUNC est posée sur un socle en téflon dans une matrice de repérage (fig. 1). Toute l'expérimentation a été effectuée de façon stérile, les évolutions des températures ont été contrôlées. Les cellules se répartissent sur le plan inférieur de la boîte NUNC où elles adhèrent. Elles sont isolées individuellement, dispersées de façon aléatoire sur les $25 \mathrm{~cm}^{2}$. Elles couvrent $4.10^{-5}$ de la surface du plan inférieur et représentent $4.10^{-8}$ du volume de 


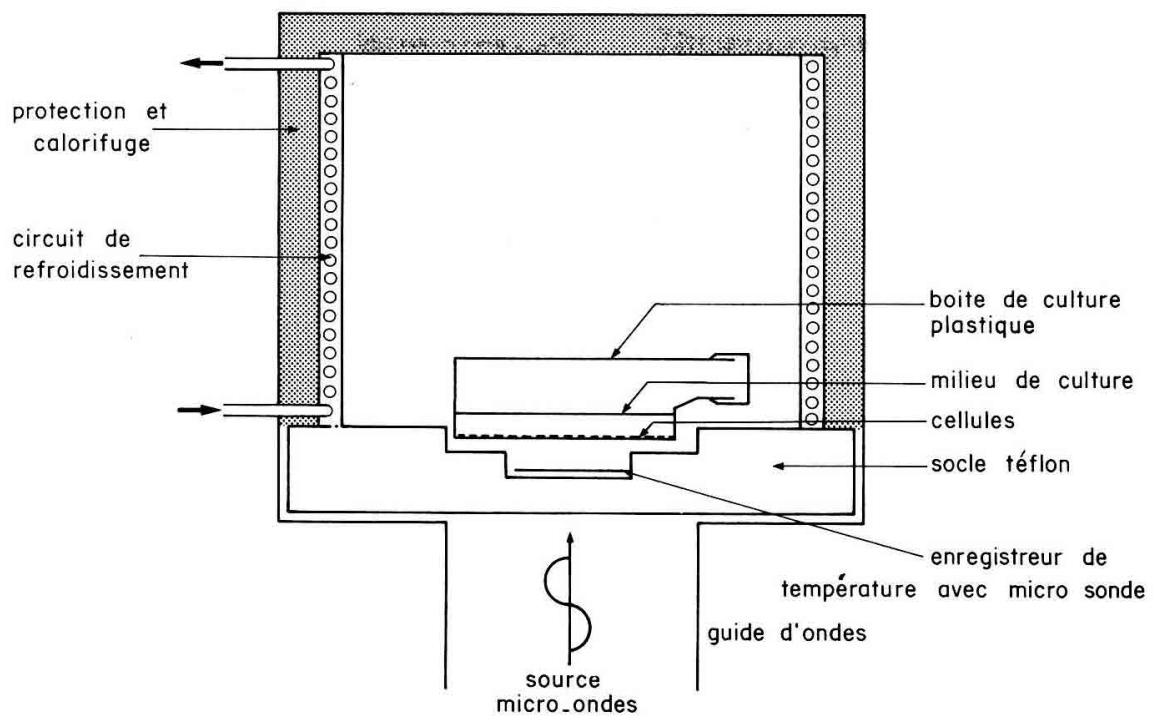

FIG. 1. - Schéma de l'applicateur.

milieu de culture au moment du traitement par les ondes électromagnétiques non ionisantes. Les temps des traitements s'étalent de 1 à $114 \mathrm{~min}$, les puissances nominales de l'émission des ondes électromagnétiques varient de 3 à $48 \mathrm{~W}$.

\section{Résultats.}

1. Effet létal des ondes électromagnétiques non ionisantes.

Une lame d'eau traversée par des ondes électromagnétiques non ionisantes capture de l'énergie sous forme de chaleur et s'échauffe. Le coefficient de capture d'énergie du milieu de culture est 4,4 fois plus grand que celui de l'eau. La température du milieu de culture s'élève plus rapidement que celle de l'eau pour une même énergie incidente (tabl: 1).

\section{TABLEAU 1}

Echauffement de l'eau, de la solution saline de Hanks et du milieu de culfure MEM. 0121 Tris. Le traitement par les micro-ondes dure $6 \mathrm{~min}$, à la puissance d'émission de $48 \mathrm{~W}$. La température est mesurée par une microsonde dans les $5 \mathrm{ml}$ de liquide mis dans la boîte de culture, au contact de la paroi inférieure.

Température dans la boîte de culture

Eau bidistillée

$33,5^{\circ} \mathrm{C}$

\begin{tabular}{ll} 
Début. $\ldots \ldots \ldots \cdots \cdots \cdots \cdots \cdots \cdots$ & $33,5^{\circ} \mathrm{C}$ \\
\hline Fin $\ldots \ldots \cdots \cdots \cdots$ & $36,5^{\circ} \mathrm{C}$
\end{tabular}

Solution saline de Hanks
Milieu de culture 121 MEM. 4S. E. Tris

$\Delta t \ldots \ldots \ldots \ldots \ldots \ldots+3^{\circ} \mathrm{C}$

$33,4^{\circ} \mathrm{C}$

$33,4^{\circ} \mathrm{C}$

$44,5^{\circ} \mathrm{C} \quad 46,0^{\circ} \mathrm{C}$

$11,5^{\circ} \mathrm{C}$

$+12,6^{\circ} \mathrm{C}$ 
La thermosensibilité des cellules est caractérisée par les relations existantes entre le taux de survie, la température ef le temps d'exposition à cette température. Les cellules sont soumises pendant des temps courts de 1 à $60 \mathrm{~min}$, à des chocs de température en plongeant les boîtes NUNC dans des bains d'eau thermostatés (fig. 2). Elles sont aussi cultivées pendant un, huit ou dix jours à des températures supra-optimales (tabl. 3). La lignée cellulaire CHO présente nettement des caractéristiques de thermosensibilité par rapport à la lignée cellulaire PK 15. Les cellules PK 15 et CHO sont sensibles à l'exposition aux ondes électromagnétiques non ionisantes (tabl. 2, 3). Le taux
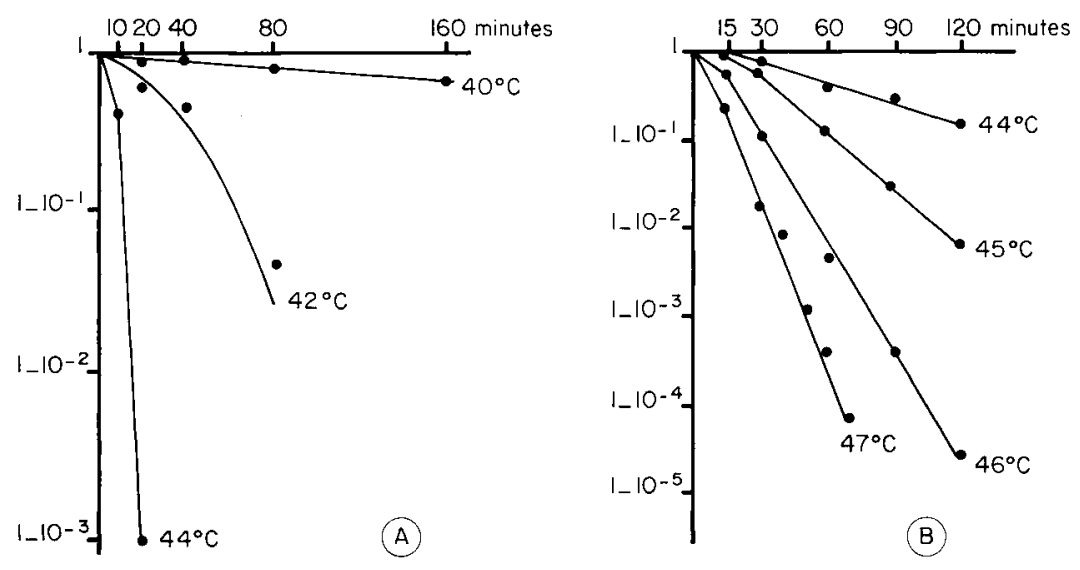

FIG. 2. - Taux de survie de la lignée cellulaire $\mathrm{CHO}$ (A) el de la lignée cellulaire PK 15 (B.). Abscisse : durée du choc thermique; ordonnée : taux de survie.

TABLEAU 2

Rapport entre le taux de survie des cellules PK 15 et $\mathrm{CHO}$ et les paramètres de l'exposition des cellules aux ondes électro-magnétiques non ionisantes émises d la puissance de $16 \mathrm{~W}$. La puissance convertie en chaleur par $\mathrm{cm}^{2}$ est de $65 \mathrm{~mW}$.

\begin{tabular}{|c|c|c|c|c|c|c|}
\hline \multirow{2}{*}{$\begin{array}{l}\text { Energie } \\
\text { d'émission } \\
\text { (en joules) }\end{array}$} & \multirow{2}{*}{$\begin{array}{c}\text { Temps } \\
\text { d'exposition } \\
\text { (en secondes) }\end{array}$} & \multicolumn{2}{|c|}{ Taux de survie $\times .100$} & \multirow{2}{*}{$\begin{array}{l}\text { Température } \\
\text { du milieu } \\
\text { en fin } \\
\text { d'exposition }\end{array}$} & \multicolumn{2}{|c|}{$\begin{array}{c}\text { Taux de survie } \times 100 \\
\text { après choc thermique } \\
\text { seul }\end{array}$} \\
\hline & & $\begin{array}{l}\text { cellules } \\
\text { PK } 15\end{array}$ & $\begin{array}{c}\text { cellules } \\
\text { CHO }\end{array}$ & & $\begin{array}{l}\text { cellules } \\
\text { PK } 15\end{array}$ & $\begin{array}{c}\text { cellules } \\
\mathrm{CHO}\end{array}$ \\
\hline $\begin{array}{r}360 \\
720 \\
1440 \\
1920 \\
2880 \\
3840 \\
5760 \\
7200 \\
7920 \\
8640 \\
15360 \\
17280 \\
30720 \\
61440\end{array}$ & $\begin{array}{c}22,30 \\
45 \\
90 \\
120 \\
180 \\
240 \\
360 \\
450 \\
495 \\
540 \\
960 \\
1080 \\
1920 \\
3840\end{array}$ & $\begin{array}{c}100 \\
\overline{-} \\
\overline{-} \\
\frac{81}{\overline{6}} \\
\frac{-}{\overline{-}} \\
\frac{36}{18} \\
<1.10^{-3}\end{array}$ & $\begin{array}{c}100 \\
\overline{-} \\
\overline{-} \\
\overline{76} \\
\overline{-} \\
\overline{65} \\
\overline{49} \\
\frac{\overline{2}}{24} \\
<1.10^{-3}\end{array}$ & $\begin{array}{l}30,4^{\circ} \mathrm{C} \\
\overline{-} \\
29,6^{\circ} \mathrm{C} \\
\overline{-} \\
29,4^{\circ} \mathrm{C} \\
30,0^{\circ} \mathrm{C} \\
30,6^{\circ} \mathrm{C} \\
30,7^{\circ} \mathrm{C} \\
33, \bar{\circ}^{\circ} \mathrm{C} \\
34,5^{\circ} \mathrm{C} \\
36,1^{\circ} \mathrm{C} \\
37,6^{\circ} \mathrm{C}\end{array}$ & $\begin{array}{l}100 \\
100 \\
100 \\
100 \\
100 \\
100 \\
100 \\
100 \\
100 \\
100 \\
100 \\
100 \\
100 \\
100\end{array}$ & $\begin{array}{l}100 \\
100 \\
100 \\
100 \\
100 \\
100 \\
100 \\
100 \\
100 \\
100 \\
100 \\
100 \\
100 \\
100\end{array}$ \\
\hline
\end{tabular}


TABLEAU 3

Rapport entre les taux de survie des cellules PK 15 et $\mathrm{CHO}$ et les paramètres de l'exposition des cellules oux ondes électromagnétiques non ionisantes émises à la puissance de $48 \mathrm{~W}$. La puissance convertie en chaleur par $\mathrm{cm}^{2}$ est de $110 \mathrm{~mW}$.

\begin{tabular}{|c|c|c|c|c|c|c|}
\hline \multirow{2}{*}{$\begin{array}{l}\text { Energie } \\
\text { d'émission } \\
\text { (en joules) }\end{array}$} & \multirow{2}{*}{$\begin{array}{c}\text { Temps } \\
\text { d'exposition } \\
\text { (en secondes) }\end{array}$} & \multicolumn{2}{|c|}{ Taux de survie $\times 100$} & \multirow{2}{*}{$\begin{array}{c}\text { Température } \\
\text { du milieu } \\
\text { en fin } \\
\text { d'exposition }\end{array}$} & \multicolumn{2}{|c|}{$\begin{array}{c}\text { Taux de survie } \times 100 \\
\text { après choc thermique } \\
\text { seul }\end{array}$} \\
\hline & & $\begin{array}{l}\text { cellules } \\
\text { PK } 15\end{array}$ & $\begin{array}{c}\text { cellules } \\
\mathrm{CHO}\end{array}$ & & $\begin{array}{l}\text { cellules } \\
\text { PK } 15\end{array}$ & $\begin{array}{c}\text { cellules } \\
\mathrm{CHO}\end{array}$ \\
\hline $\begin{array}{r}360 \\
720 \\
1440 \\
2880 \\
5760 \\
7200 \\
7920 \\
8640 \\
11520 \\
17280\end{array}$ & $\begin{array}{l}7,50 \\
15 \\
30 \\
60 \\
120 \\
150 \\
165 \\
180 \\
240 \\
360\end{array}$ & $\begin{array}{r}98 \\
88 \\
87 \\
80 \\
68 \\
48 \\
35 \\
24 \\
<-1.10^{-2}\end{array}$ & $\begin{array}{c}100 \\
93 \\
88 \\
82 \\
56 \\
\overline{-} \\
\overline{27} \\
<\overline{1.10^{-2}}\end{array}$ & $\begin{array}{c}31^{\circ} \mathrm{C} \\
-\bar{C} \\
32,0^{\circ} \mathrm{C} \\
34,6^{\circ} \mathrm{C} \\
35,7^{\circ} \mathrm{C} \\
36 \bar{\circ}^{\circ} \mathrm{C} \\
39,6^{\circ} \mathrm{C} \\
42,3^{\circ} \mathrm{C}\end{array}$ & $\begin{array}{l}100 \\
100 \\
100 \\
100 \\
100 \\
100 \\
100 \\
100 \\
100 \\
100\end{array}$ & $\begin{array}{r}100 \\
100 \\
100 \\
100 \\
100 \\
100 \\
100 \\
100 \\
100 \\
90\end{array}$ \\
\hline
\end{tabular}

de survie décroît avec l'augmentation de l'énergie incidente. Pour des puissances de $48 \mathrm{~W}$ ou de $16 \mathrm{~W}$ et des niveaux d'énergie incidente respectivement de $17280 \mathrm{~J}$ ou de $61400 \mathrm{~J}$ le taux de survie cellulaire est inférieur à $1.10^{-4}$. Il y a donc mort de toutes les cellules. Les températures atteintes à la fin du traitement sont respectivement de $42,3{ }^{\circ} \mathrm{C}$ après $6 \mathrm{~min}$ et de $37,6^{\circ} \mathrm{C}$ après $64 \mathrm{~min}$. Or il faut rappeler, pour les cellules PK 15, que des chocs thermiques de $42,3^{\circ} \mathrm{C}$ pendant 10 min sont sans effet, qu'après $24 \mathrm{~h}$ d'exposition à $42,3^{\circ} \mathrm{C}$ les cellules PK 15 ont encore un taux de survie de 0,45 et qu'après

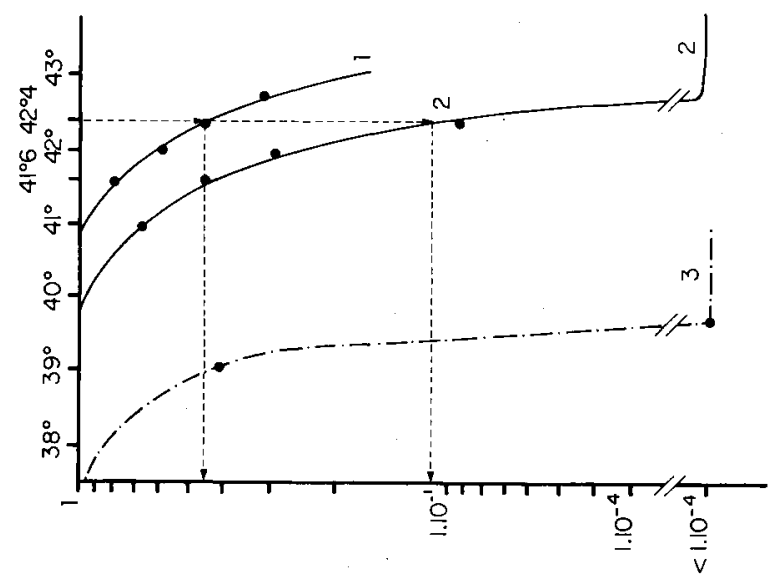

FIG. 3. - Taux de survie des lignées cellulaires PK $15(1,2)$ ef CHO (3) exposées à des tempérarures supraoptimales. Courbe 1 , exposition de $24 \mathrm{~h}$ aux températures supra-optimales. Courbes 2 et 3 , exposition de 8 jours aux températures supra-optimales. Abscisse : températures; ordonnée : taux de survie. 
8 jours d'exposition à $42,3^{\circ} \mathrm{C}$ elles ont un taux de survie de 0,1 . L'effet thermique induit par les ondes électromagnétiques non ionisantes ne peut expliquer à lui seul le faux de survie très bas des cellules PK 15. Bien que les cellules $\mathrm{CHO}$ soient plus thermosensibles que les cellules PK 15, la même conclusion apparaît dans le cas des émissions faites à $48 \mathrm{~W}$, puisque des cellules $\mathrm{CHO}$ exposées pendant $10 \mathrm{~min}$ d̀ $42,3{ }^{\circ} \mathrm{C}$ ont un taux de survie de 0,80 très supérieur à $1 \cdot 10^{-2}$. Cette conclusion s'affirme beaucoup plus fortement lorsque la puissance d'émission est de $16 \mathrm{~W}$. Dans ce cas la température du milieu de culture atteint $37,6^{\circ} \mathrm{C}$ en $64 \mathrm{~min}$. C'est une te mpérature compatible avec la vie des cellules PK 15 et CHO. Pourtant après 64 min d'exposition les deux lignées cellulaires ont un taux de survie de $1.10^{-2}$. Enfin les courbes des taux de survie de PK 15 et de CHO traitées par les ondes électromagnétiques non ionisantes se recouvrent. Les mécanismes de la sensibilité aux ondes électromagnétiques sont donc différents de ceux responsables de la thermosensibilité.

2. Effet des ondes électromagnétiques sur la mufagenèse.

Le pouvoir mulagène des ondes électromagnétiques non ionisantes a été testé par rapport à celui d'un agent alkylant, l'EMS. L'interaction entre la mutagenèse à I'EMS et une exposition aux ondes électromagnétiques non ionisantes a été étudiée. Le pouvoir mutagène est mesuré par la fréquence des colonies mutantes isolées après sélection à la thioguanine $\left(8 \cdot 10^{-6} \mathrm{M}\right)$ ou à l'ouabaïne $\left(1 \cdot 10^{-6} \mathrm{M}\right)$. Ces deux résistances apparaissent lorsqu'un seul gène a muté. La résisłance à la thioguanine (TGr) a une localisation liée au sexe, elle est récessive. La résistance à l'ouabaïne (OUAr) a une localisation autosomale, elle est codominante. Les résultats expérimentaux des mutagenèses sont présentés dans les tableaux 4 et 5 . Le traitement à l'EMS a un pouvoir mutagène en accord avec tous les résultats connus tant pour la résistance à la thioguanine que pour la résistance à l'ouabaïne. L'exposition aux ondes électromagnétiques non ionisantes de $16 \mathrm{~W}$ pendant 15 min dans un tampon de Hanks n'a aucun pouvoir mutagène. Mais, cette même exposition appliquée après la mutagenèse amplifie le pouvoir mutagène de l'EMS par un facteur d'ordre 3.

TABLEAU 4

Souche cellulaire $P K 15 C_{2}$. Thioguanine résistance, expression récessive, situation liéc au sexe

\begin{tabular}{|c|c|c|c|c|}
\hline & Témoin & $\begin{array}{l}\text { E.M.S. } \\
300 \mu \mathrm{g} / \mathrm{ml} \\
6 \mathrm{~h}\end{array}$ & $\begin{array}{l}\text { E.M.S. } \\
300 \mu \mathrm{g} / \mathrm{ml} \\
6 \mathrm{~h}+\mathrm{M} . \mathrm{O} . \\
16 \mathrm{~W} 15 \mathrm{~min}\end{array}$ & $\begin{array}{l}\text { Traitement } \\
\text { M.O. } \\
16 \text { W15 min }\end{array}$ \\
\hline $\begin{array}{l}\text { Nombre de répétitions indépendantes } \ldots \\
\text { Nombre de cellules mutagénéisées à }\end{array}$ & 5 & 5 & 5 & 5 \\
\hline $\begin{array}{l}\text { chaque mutagenèse } \ldots \ldots \ldots \ldots \ldots \ldots \\
\text { Nombre total de boîtes étalées.......... } \\
\text { Nombre de cellules par boîte } \ldots \ldots \ldots \ldots \\
\text { Coefficients de correction dus aux taux }\end{array}$ & $\begin{array}{l}10.10^{6} \\
31 \\
5.10^{5}\end{array}$ & $\begin{array}{l}10.10^{6} \\
10 \\
5.10^{5}\end{array}$ & $\begin{array}{l}10.10^{6} \\
10 \\
5.10^{5}\end{array}$ & $\begin{array}{l}10.10^{6} \\
19 \\
5.10^{5}\end{array}$ \\
\hline $\begin{array}{c}\text { de survie } \ldots \ldots \ldots \ldots \ldots \ldots \ldots \ldots \ldots \\
\text { Fréquence moyenne des mutants pour }\end{array}$ & 0,35 & 0,35 & 0,35 & 0,35 \\
\hline $1.10^{6}$ cellules $\ldots \ldots \ldots \ldots \ldots \ldots$ & 2,34 & 12,25 & 32,46 & 1,49 \\
\hline
\end{tabular}


TABLEAU 5

Souche cellulaire PK $15 C_{2}$. Ouabaïne résistance, expression génique codominante, situation outosomale

\begin{tabular}{|c|c|c|c|c|}
\hline & Témoin & $\begin{array}{c}\text { E.M.S. } \\
300 \mu \mathrm{g} / \mathrm{ml} \\
6 \mathrm{~h}\end{array}$ & $\begin{array}{c}\text { E.M.S. } \\
300 \mu \mathrm{g} / \mathrm{ml} \\
6 \mathrm{~h} \text { suivi } \\
\text { du traitement } \\
\text { M.O. } \\
16 \mathrm{~W} 15 \mathrm{~min}\end{array}$ & $\begin{array}{l}\text { Traitement } \\
\text { M.O. } \\
16 \text { W } 15 \text { min }\end{array}$ \\
\hline \multirow{4}{*}{$\begin{array}{l}\text { Nombre de répétitions indépendantes } \\
\text { Nombre de cellules mutagénéisées à } \\
\text { chaque mutagenèse. } \ldots \ldots \ldots \ldots \ldots \ldots \\
\text { Nombre total de boîtes étalées } \ldots \ldots \ldots \ldots \\
\text { Nombre de cellules par boîtes } \ldots \ldots \ldots \\
\text { Coefficients de correction dus aux taux } \\
\text { de survie } \ldots \ldots \ldots \ldots \ldots \ldots \ldots \ldots \\
\text { Fréquence moyenne des mutants pour } \\
1.10^{6} \text { cellules } \ldots \ldots \ldots \ldots \ldots \ldots \ldots \ldots\end{array}$} & 5 & 5 & 5 & 5 \\
\hline & $\begin{array}{l}10.10^{6} \\
28 \\
5.10^{5}\end{array}$ & $\begin{array}{l}10.10^{6} \\
22 \\
5.10^{5}\end{array}$ & $\begin{array}{l}10.10^{6} \\
24 \\
5.10^{5}\end{array}$ & $\begin{array}{l}10.10^{6} \\
16 \\
5.10^{5}\end{array}$ \\
\hline & 0,35 & 0,35 & 0,35 & 0,35 \\
\hline & 0,80 & 11,76 & 43,71 & 0,69 \\
\hline
\end{tabular}

\section{Discussion.}

\section{Caractéristiques de l'instrumentation.}

Une instrumentation ayant les mêmes objectifs d'utilisation que la nôtre a été conçue et réalisée par Chen ef Lin $(1975,1976)$. Les cellules trypsinées y sont placées dans la lumière d'une seringue. Nous avons mis au point un autre type d'instrumentation permettant d'exposer aux actions des ondes électromagnétiques non ionisantes les cellules fixées sur leur support et baignant dans leur milieu de culture. L'intérêt de cette instrumentation est de contrôler la température du milieu de culture par un système de refroidissement, et de maintenir cette température dans les limites favorables à la vie cellulaire. Les mesures des températures réalisées en fonction du temps permettent d'estimer à 48 ef $110 \mathrm{~mW}$ par $\mathrm{cm}^{2}$ l'énergie captée (voir annexe). Dans notre système instrumental les cellules sont réparties aléatoirement sur la surface de culture, ont des membranes intactes, ne sont pas en phase d'attente avant la croissance, sont séparées les unes des autres, ce qui évite toute interaction ef sont placées sur le lieu où leurs descendants formeront une colonie. Ces caractéristiques expérimentales permettent d'exposer des cellules intactes, de quantifier les réponses biologiques ef d'utiliser les méthodologies de la génétique somatique.

2. Séparation des effets thermiques et des effets spécifiques.

Notre système expérimental met en évidence les effets spécifiques qui précèdent les effets thermiques dus à l'échauffement induit par les ondes électromagnétiques dans le milieu de culture et les cellules. La fraction d'énergie captée par les molécules du milieu de culture et par les macromolécules est partagée en deux parties. L'une sert à l'élévation de la température du milieu de culture et des cellules, l'autre est évacuée par la source froide (voir annexe). La somme de ces deux parties caractérise la capacité de capture d'énergie des ions, des molécules et des macromolécules. 
L'énergie des ondes électromagnétiques délocalise les électrons de ces ions, molécules et macromolécules, entraînant la déstabilisation de leur conformation probablement par un mécanisme d'amplification quantique (Bawin ef al., 1978). Cette délocalisation induite par les champs électromagnétiques a pour conséquence l'augmentation du nombre des électrons libres et de leurs vitesses. La température du milieu de culture repère ce niveau d'agitation électronique. Le problème est de montrer que la température du milieu, mesurée pendant l'exposition aux ondes électromagnétiques est aussi celle des cellules. L'équilibre des températures entre le milieu de culture et les cellules est afteint immédiatement pour deux raisons. La première est que la surface d'échange est maximale entre les 1000 cellules qui sont dispersées et isolées sur $25 \mathrm{~cm}^{2}$. ef qui représentent $4 \cdot 10^{-8}$ du volume du milieu de culture. La seconde est que les chaleurs spécifiques du milieu de culture et des cellules calculées selon la loi de Dulong et Petit sont équivalentes $\left(4,39.10^{7} \mathrm{ergs} / \mathrm{g} /{ }^{\circ} \mathrm{C}\right)$.

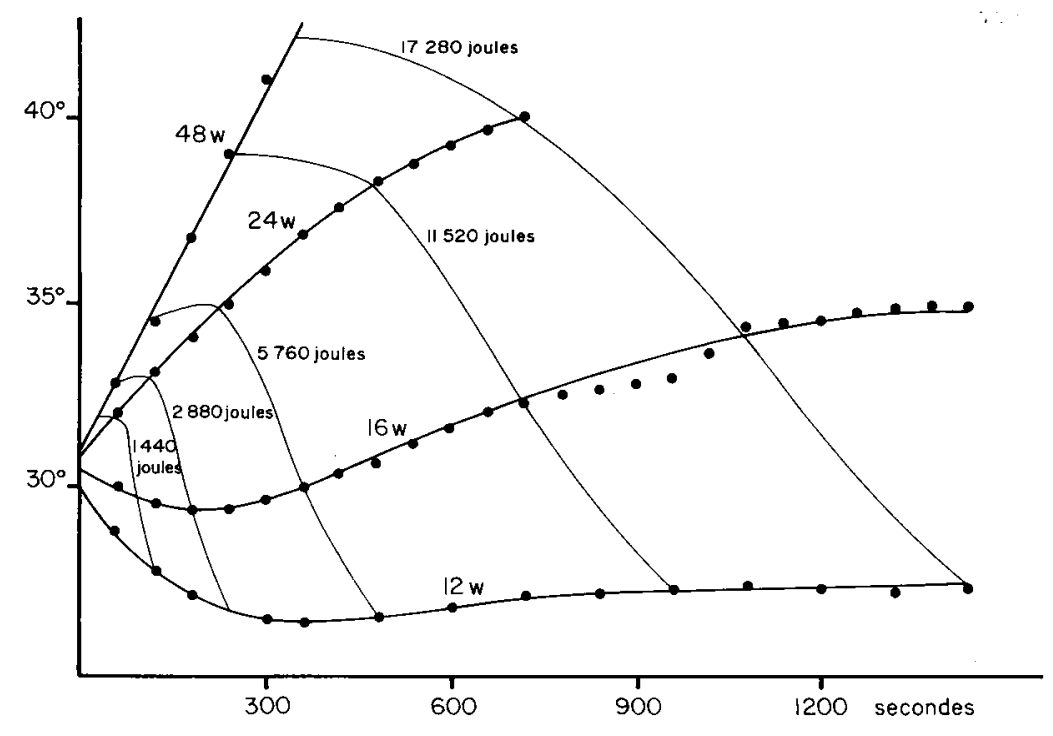

FIG. 4. - Evolution des températures dans $5 \mathrm{ml}$ de milieu MEM 0121 supplémenté avec 4 p. 100 de sérum, réparti dans une boite falcon de $25 \mathrm{~cm}^{2}$. Les puissances d'émission sont $12 \mathrm{~W}, 16 \mathrm{~W}, 24 \mathrm{~W}, 48 \mathrm{~W}$. Abscisse : temps en secondes ; ordonnée : température.

\section{Existence de la spécificité des effets.}

Dans ces conditions expérimentales il ne devrait pas y avoir de différence entre les cinétiques de thermosensibilité que l'échauffement soit obłenu par conductance thermique ou application d'ondes électromagnétiques non ionisantes, si ces dernières n'avaient pas d'effets spécifiques. Or, pour des durées égales d'exposition aux mêmes températures les taux de survie mesurés en présence d'ondes électromagnétiques non ionisantes sont toujours plus faibles. En particulier, l'exposition aux ondes électromagnétiques non ionisantes à des températures optimales $\left(37\right.$ à $38^{\circ} \mathrm{C}$ ) est létale. Pour des durées égales d'exposition aux mêmes températures, les taux de survie de deux lignées cellulaires possédant des thermosensibilités différentes, sont égaux. La 
mortalité induite par les ondes électromagnétiques non ionisantes est due à des mécanismes différents de ceux responsables de la thermosensibilité. Des effets non thermiques ont déjà été décrits, tels que les réponses tératogènes du développement des pupes de Tenebrio molitor (Carpenter et Livstone, 1971), ou des modifications de la morphologie cellulaire (Lindaver et al., 1974 ; Lin ef Chen, 1975 ; Sawicki et Ostrowski, 1968 ; Stodolinik-Barnaska, 1967 ; Valtonen, 1966 ; Chen et Lin, 1976). Mais ces résultats utilisent comme critère significatif la seule température du milieu dans lequel les cellules baignent. Nous avons ajouté deux autres critères de décision. L'un est la comparaison entre la thermosensibilité d'une lignée cellulaire et sa sensibilité aux ondes électromagnétiques non ionisantes, et cela indépendamment pour les deux lignées cellulaires PK 15 et CHO. L'autre est la comparaison des sensibilités respectives aux ondes électromagnétiques non ionisantes de deux lignées cellulaires ayant des thermosensibilités distinctes. De plus, nous avons utilisé comme réponse expérimentale la mortalité cellulaire ce qui permet à partir des abaques obtenues de définir les conditions de sélection de mutants résistants ou sensibles aux ondes électromagnétiques non ionisantes.

\section{Effet mutagène.}

Les ondes électromagnétiques non ionisantes n'ont aucun pouvoir mutagène sur les cellules de mammifères. Ce résultat est en accord avec ceux acquis pour E. coli (Averbeck ef al., 1976 ; Bawin ef al., 1978 ; Webb, 1975) et pour Saccharomices et Aspergillus nidulans (Averbeck ef al., 1976 ; Baranski et al., 1976). Par confre, ce qui est plus nouveau est la description de l'effet multiplicateur des ondes électromagnétiques non ionisantes sur le pouvoir mutagène de l'EMS. L'EMS est un agent alkylant qui greffe un radical $\mathrm{C}_{2} \mathrm{H}_{5}$ très généralement à une guanine (Freeze, 1971) et, ainsi, cause de petites distorsions de l'ADN. Les mutations représentent la partie de ces petites distorsions qui n'ont pas été corrigées au moment où s'accomplit la réplication de l'ADN. Cette partie est augmentée, soił parce que les enzymes de réparation fonctionnent mal comme dans le Xeroderma pigmentosum (Cleaver et Bootsma, 1975), soit parce qu'elles ont un accès à l'ADN devenu difficile sans que leurs capacités de réparation aient été modifiées, soit parce que les alkylations sont en plus grand nombre.

Il est improbable que les ondes électromagnétiques non ionisantes augmentent le nombre de bases alkylées puisque les cellules sont soumises à leur action après un lavage au Hanks éliminant l'EMS. Les ondes électromagnétiques non ionisantes pourraient diminuer l'efficacité des enzymes de correction en modifiant soit la structure du DNA, soit celle de la chromatine, soit celle des enzymes elles-mêmes. Neumann et Katchalsky (1972) ont montré que les doubles hélices de poly (A)-2 poly (U) sont dissociées en deux brins par des champs électromagnétiques qui déplacent l'atmosphère ionique proche, libérant les forces de répulsion entre les brins antiparallèles. Ceci cause la dissociation des paires de bases UA et si le $\mathrm{pH}$ est légèrement acide les résidus adénine découplés sont rapidement protonés, ce qui gêne la réassociation. Chiabrera et al. (1979) ont montré que les chromosomes des cellules de globules rouges d'amphibien soumis à des champs électromagnétiques perdent certaines associations stéréospécifiques entre l'ADN et les protéines, ce qui se traduit par une augmentation du nombre des sites d'intercalation de l'acridine orange dans l'ADN dénudé des chromosomes. Ces deux mécanismes, modification de la structure double hélice et empê- 
chement des associations stéréospécifiques entre l'ADN et les protéines ou bien entre les protéines pourraient être à l'origine de l'augmentation du nombre des sites alkylés non corrigés au moment de la réplication de l'ADN, entraînant une augmentation de la fréquence des mutations induites.

Reçu en octobre 1979.

Accepté en mai 1980.

\section{ANNEXE}

Analyse thermodynamique du Système

Une boîte "NUNC » fermée, remplie de $5 \mathrm{ml}$ de milieu de culture s'échauffe au cours de son exposition aux ondes électromagnétiques non ionisantes. Cet échauffement est d'autant plus rapide que la puissance d'émission est grande. Les abaques relevées (fig. 4) permettent une étude des cinétiques des températures et une analyse thermodynamique du système. Entre les instants $t$ et $t+\Delta t$ la variation de la quantité de chaleur de la boîte de culture cellulaire s'écrit :

$$
\Delta q_{1}=\varphi_{1} \Delta t+\lambda\left(T_{1}-T_{2}\right) \Delta t
$$

où $\varphi_{1}$ est l'énergie capturée sous forme de chaleur par le milieu de culture, $\lambda$ la conductance thermique entre le milieu de culture, de température $T_{1}$ est le circuit réfrigérant de température $T_{2}$.

Cette énergie $\Delta q_{1}$ est aussi égale au produit de la masse du milieu $\left(m_{1}=5 \mathrm{~g}=5.10^{-3} \mathrm{~kg}\right)$, par la chaleur spécifique du milieu $\left(C_{1}=1,05 \mathrm{cal} / \mathrm{g} /{ }^{\circ} \mathrm{C}=4,4.10^{3} \mathrm{~J} . \mathrm{kg}^{-1} \mathrm{oK}-1\right)$ et par la variation de température du milieu $\Delta T_{1}$ :

$$
\Delta q_{1}=m_{1} C_{1} \Delta T_{1} .
$$

On écrit de même les échanges de chaleur du circuit de refroidissement avec le milieu de culture, la source froide, de température $T_{3}$, et, éventuellement la source de radiations :

$$
\begin{aligned}
\Delta q_{2} & =\varphi_{2} \Delta t+\lambda\left(T_{1}-T_{2}\right) \Delta t-\mu\left(T_{2}-T_{3}\right) \Delta t \\
& =m_{2} C_{2} \Delta T_{2}
\end{aligned}
$$

On peut également envisager que la source froide, mal thermostatée voit sa température dériver dans le temps. Il faut alors écrire, de façon analogue :

$$
\begin{aligned}
\Delta q_{3} & =\varphi_{3} \Delta t+\mu\left(T_{2}-T_{3}\right) \Delta t \\
& =m_{3} C_{3} \Delta T_{3}
\end{aligned}
$$

où $\varphi_{3}$ désigne la puissance reçue par la source froide de la part du milieu ambiant, et supposée constante pendant la durée d'une expérience. Ces équations se traduisent par le système suivant d'équations différentielles :

$$
\begin{gathered}
\frac{d T_{1}}{d t}=\frac{\varphi_{1}}{m_{1} C_{1}}-\frac{\lambda}{m_{1} C_{1}} T_{1}+\frac{\lambda}{m_{1} C_{1}} T_{2} \\
\frac{d T_{2}}{d t}=\frac{\varphi_{2}}{m_{2} C_{2}}+\frac{\lambda}{m_{2} C_{2}} T_{1}-\frac{\lambda+\mu}{m_{2} C_{2}} T_{2}+\frac{\mu}{m_{2} C_{2}} T_{3} \\
\frac{d T_{3}}{d t}=\frac{\varphi_{3}}{m_{3} C_{3}}+\frac{\mu}{m_{3} C_{3}} T_{2}-\frac{\mu}{m_{3} C_{3}} T_{3} .
\end{gathered}
$$

En remarquant que $m_{1} C_{1} \ll m_{2} C_{2} \ll m_{3} C_{3}$, et qu'à l'instant initial, $T_{2}(0)=T_{3}(0)$, ce système conduit à la solution suivante, pour la température $T_{1}$ du milieu de culfure :

$$
T_{1}(t)=\alpha+\beta t+\gamma \mathrm{e}^{-r t}+\delta \mathrm{e}^{-s t}
$$


où :

$$
\begin{aligned}
& r=\frac{\lambda}{m_{1} C_{1}} ; s=\frac{\mu}{m_{2} C_{2}} \quad(r>s) ; \\
& \alpha=\frac{\varphi_{1}}{\lambda}+\frac{\varphi_{2}}{\mu}+T_{3}(0)-\beta\left(\frac{m_{1} C_{1}}{\lambda}+\frac{m_{2} C_{2}}{\mu}\right) ;
\end{aligned}
$$

avec :

$$
\begin{aligned}
& \beta=\frac{\varphi_{1}+\varphi_{2}+\varphi_{3}}{m_{1} C_{1}+m_{2} C_{2}+m_{3} C_{3}} ; \\
& \gamma=T_{1}(0)-T_{3}(0)-\frac{\varphi_{1}}{\lambda}+\left(\frac{\varphi_{2}}{m_{2} C_{2}}-\beta \frac{\mu}{\varphi} \frac{m_{1} C_{1}}{m_{2} C_{2}}\right)\left(\frac{\varphi_{1}}{m_{1} C_{1}}-\frac{\mu}{m_{2} C_{2}}\right)^{-1} \\
& \delta=\frac{\lambda m_{2} C_{2}}{\mu m_{1} C_{1}}\left(\frac{\varphi_{2}}{m_{2} c_{2}}-\beta\right)\left(\frac{\varphi_{1}}{m_{1} C_{1}}-\frac{\mu}{m_{2} C_{2}}\right)^{-1} .
\end{aligned}
$$

Les données des abaques ont été utilisées pour estimer les paramètres de ces équations, c'est-à-dire les quantités : $r, s, \beta, \frac{\varphi_{1}}{m_{1} C_{1}}, \frac{\varphi_{2}}{m_{2} C_{2}}$, et les températures initiales $T_{3}(0)$ non mesurées.

On a supposé, ce qui paraissait légitime, que la puissance $\varphi_{1}$ convertie en chaleur était proportionnelle à la puissance émise $W$, soit :

$$
\varphi_{1}=W \psi_{1}, \varphi_{2}=W \psi_{2} \text {, et cherché à estimer les coefficients } \psi_{1} \text { et } \psi_{2} \text {. }
$$

Le programme d'estimation est dérivé à l'algorithme de Marquard d'ajustements non linéaires. L'ajustement ne s'avère correct qu'en excluant les deux expériences correspondant à la puissance d'émission de $48 \mathrm{~W}$, el fournit une valeur de $10 \mathrm{p} .100$ au coefficient $\psi_{1}$. Les puissances converties en chaleur dans les trois séries d'expériences à 12,16 et $24 W$ d'émission sont ainsi évaluées à 48,64 et $96 \mathrm{~mW} / \mathrm{cm}^{2}$. Pour la puissance d'émission de $48 \mathrm{~W}$, le même coefficient $\psi_{1}$ ne peut rendre compte de la proportion d'énergie convertie en chaleur ; en utilisant les autres valeurs estimées, on peut évaluer la valeur de la puissance $\varphi_{1}$ d'après les pentes à l'origine des abaques, et le coefficient $\psi_{1}$ correspondant vaut environ 5,7 p. 100 , et correspond à une puissance convertie en chaleur de $110 \mathrm{~mW} / \mathrm{cm}^{2}$. La moindre efficacité de l'émission à $48 \mathrm{~W}$ pourrait être due à un phénomène de réflexion des ondes sur les surfaces traversées ou la saturation des capteurs. Ces valeurs numériques doivent être considérées seulement comme des ordres de grandeur étant donné les approximations faites dans l'analyse par rapport au nombre de paramètres à estimer.

\section{Références}

ALAM M. I., BARTHAKUR N., LAMBERT N. G., KASATIYA S. S., 1978. Cytological effects of microwave radiation in chinese hamster cells in vifro. Can. J. Genet. Cytol., 20, 23-30.

AVERBECK D., DARDALHON M., BERTAUD A. J., 1976. Microwaves action in procaryotic and eucaryotic cells and a possible interaction with X-rays. J. Microwave Power, 11, 143-144.

BARANSKI S., EDELWEJN Z., 1975. Experimental morphologic and electroencephalographic studies of microwave effects on the nervous system. Ann. N. Y. Acad. Sci., 247, 109-116.

BARANSKI S., DEBIEC H., KWARECKI K., MEZYKOWSKY T., 1976. Influence of microwaves on genetical processes of Aspergillus nidulans. J. Microwave Power, 11, 146-147 (abstr. 3A).

BAWIN S. M., SHEPPARD A., ADEY W. R., 1978. Possible mechanisms of weak electromagnefic field coupling in brain tissue. Bioelectrochem. Bioenerg., 5, 67-76.

BERTEAUD A. J., DARDALHON M., REBEYROTTE N., AVERBECK D., 1975. Action d'un rayonnement électromagnétique à longueur d'onde millimétrique sur la croissance bactérienne. C. R. Acad. Sci. Paris, sér. D, 281, 843-846.

CARPENTER R. L., LIVSTONE E. M., 1971. Evidence for non thermal effects of microwave radiation : abnormal development of irradiated insect pupae. IEEE Trans. Microwave Theor. Tech. 19, 173-178. 
CHEN K. C., LIN C. J., 1976. Cytotoxic effects of electromagnetic radiation on chinese hamster cells in culture. J. Microwave Power, 11, 140-141.

CHIABRERA A., HINSENKAMP M., PILLA A. A., RYABY J., PONTA D., BELMONT A., BELTRAME F., GRATAROLLA M., NICOLINI C., 1979. Cytofluorometry of electromagnetically controled cell dedifferentiation. J. Hisfochem. Cytochem., 27, 375-381.

CLEAVER J. E., BOOTSMA D., 1975. Xeroderma pigmentosum : biochemical and genetic characteristics. Ann. Rev. Gen., 9, 19-38.

FREEZE E., 1971. Molecular mechanisms of mutations. In HOLLAENDER A., Chemical mutagens. Plenum Press N. Y., 1, 1-56.

FRÖHLICH H., 1973. Low frequency vibrations of macromolecules. Physics Lett., 44A, 385.

FRÖHLICH H., 1977. Possibilities of long and short range electric interactions of biological systems. Neurosci. Res. Prog. Bull., 15, 67-72.

FRÖLICH H., 1978. Coherent electric vibrations in biological system and the cancer problem. IEEE Trans. Microwave Theor. Tech., 26, 613-617.

GRODSKY I. T., 1975. Possible physical substrates for the interaction of electromagnetic fields with biologic membranes. Ann. N. Y. Acod. Sci., 247, 117-124.

GRODSKY I. T., 1976. Neuronal membrane : a physical synthesis. Math. Biosci., 28, 191-219.

KATCHALSKY A., 1974. Dynamic patterns of brain cell assemblies. Neurosci. Res. Prog. Bull., 12, 11-187.

LIN J. C., CHEN K. C., 1975. Effects of microwave radiation on mammalian cells in vitro. Proc. USNC/URSI, 132

LINDAUER G. A., LIU L. M., SKEWES G. W., ROSENBAU M. F. J., 1974. Further experiments seeking evidence of nonthermal biological effects of microwave radiation. IEEE Trans. Microwave Theor. Techn., 8, 790-793.

MARHA K., MUSIL J., TUHA M., 1971. Electromagnefic fields and the life environment. San Francisco Press Inc.

MICHAELSON M., HOUK W. M., LEBDA N. J. A., SHIN TSU LU, MAGIN R. L., $1975 . \quad$ Biochemical and neuroendocrine aspects of exposure to microwaves. Ann. N. Y. Acad. Sci., 247, 21-47.

NEUMANN E., KATCHALSKY A., 1972. Long-lived conformation changes induced by electric impulses in biopolymeres. Proc. Nat. Acad. Sci. U. S. A., 69, 993-997.

NEUMANN E., NACHMANSOHN D., KATCHALSKY A., 1973. An attempt at an integral of nerve excitability. Proc. not. Acod. Sci. U. S. A., 70, 727-731.

PRESMANN A. S., 1970. Effect of electromagnetic fields on neurohumorol regulation in entire organisms. Electromagnefic fields and life. Plenum Press N. Y., London.

PUCK T. T., CIERCIVRA S. J., ROBINSON A., 1958. Genetics of somatic mammalian cells. J. exp. Med., 108, 945-956.

RUDDLE F. H., 1961. Chromosome variation in cell populations derived from pig kidney. Cancer Res., 21, 885-894.

SAWICKI W., OSTROWSKI K., 1968. Nonthermal effect of microwave radiation of cell in vitro on peritoneal mast cells of the rat. Am. J. Phys. Med., 17, 225-232.

STODOLINIK-BARNASKA W., 1967. Lymphoblastoid transformation of lymphocytes in vitro after microwave irradiation. Nature, 214, 102-103.

VALTONEN E. J., 1966. Giant mast cells. A special degenerative form produced by microwave radiation. Exp. Cell Res., 43, 221-224.

WEBB S. J., 1975. Genetic continuity and metabolic regulation as seen by the effects of various microwave and black light frequencies on the phenomena. Ann. N. Y. Acod. Sci., 247, 327-351. 